\title{
Las áreas del periodismo jurídico. Desarrollo y evolución en Abc, El País y Cinco Dias (1981-2011)
}

institucional.us.es/ambitos/

\section{María José Antich Gil}

Universidad Cardenal Herrera CEU

antich.marajos@gmail.com

Resumen

La información jurídica ocupa un espacio importante en la prensa actualmente. Aunque siempre se ha vinculado con la sección de Sucesos y Tribunales, la información jurídica se mueve en todas las secciones: Política, Economía, Comunicación, Cultura, Deportes, etc. Desde esta investigación se pretende establecer cuáles son las áreas del periodismo jurídico, y el desarrollo y evolución de estas áreas en Abc y El País, como generalistas, y Cinco Días, como económico. La muestra abarca los años de 1981 hasta 2011, ambos inclusive. Con este análisis

se pretende demostrar que existen tres áreas principalmente, donde el periodismo jurídico tiene mayor acogida: Política, Economía y Sociedad.

\section{Palabras clave}

Periodismo jurídico, información jurídica, derecho e información.

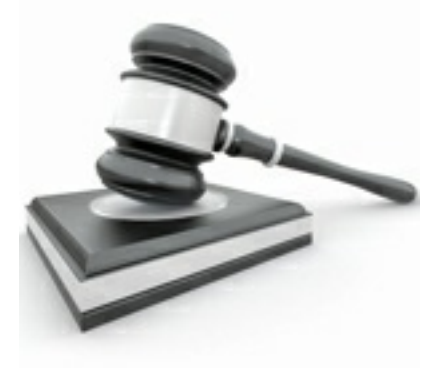

The legal information occupies an important space in the press today. Although it has always been linked to the events section and tribunals, legal information moves in all sections: Politics, Economics, Communication, Culture, Sports, etc. Since this research is to establish which areas of legal journalism and the development and evolution of these areas Abc and El País, as generalists, and Cinco Días, as economic. The sample covers the years 1981 to 2011 included. This analysis seeks to show that there are three main areas where the host has more legal journalism: Politics, Economics and Society.

\section{Keywords}

Legal journalism, legal information, law and information.

\section{INTRODUCCIÓN}

La información de Sucesos y Tribunales ha estado vinculada directamente al periodismo jurídico, por ello, desde esta investigación se pretende demostrar que tanto los sucesos como los tribunales son un área más dentro de este tipo de periodismo y no el periodismo jurídico en sí.

Es necesario manifestar que la información jurídica se mueve en todas las secciones de un periódico, pudiendo aparecer en Política, Economía, Deportes, Cultura, etc. Por ejemplo, el caso del deportista Oscar Pistorius, juzgado por asesinar a su novia, podría ir en Deportes pero también en Sociedad o Nacional.

Principalmente se trata de establecer cuáles son las áreas o los bloques temáticos del periodismo jurídico, que características tienen y como se han desarrollado y han evolucionado estas áreas entre 1981 y 2011 , años de plena democracia en España, donde esta información adquiere especial relevancia, aunque en los últimos años ha tomado impulso, sobre todo por los numerosos casos de corrupción de políticos y banqueros que han ido saliendo a la luz.

Existen diferencias importantes entre la prensa generalista y la especializada, en este caso la económica, algo que se intenta reflejar en este trabajo. La prensa económica incluye mucha información jurídica pero sus áreas nada tienen que ver con las de la prensa generalista, por ello, cabe establecer sus diferencias. 
Con la creación de la Constitución Española de 1978, el país se estructura en comunidades autónomas, gozando estas de poderes legislativos y ejecutivos propios, con sus Estatutos de Autonomía y sus competencias. Es en este momento es cuando se crean los Tribunales Superiores de Justicia, que son órganos jurisdiccionales integrados en el Poder Judicial de España, y cuyo ámbito territorial abarca una comunidad autónoma, algo que hasta entonces no existía en España. Esta organización territorial en el Estado español se configura mediante municipios, provincias y las comunidades autónomas (1).

La renovación del Poder Judicial cuya potestad sobre los derechos de los ciudadanos y de control de los gobernantes gana importancia, permite la posibilidad de asistir a los juicios y tener acceso a información que antes estaba más restringida, incitando el interés de los medios de comunicación por este tipo de información (2).

\section{OBJETIVOS E HIPÓTESIS}

Nuestra investigación persigue una serie de objetivos:

- En primer lugar indicar que la información jurídica puede moverse en todas las secciones de un periódico, y no solo tiene que aparecer en tribunales o sucesos.

- En segundo lugar, demostrar que existen tres áreas principalmente en el periodismo jurídico, y son Sociedad, Economía y Política.

- En tercer lugar, señalar las diferencias entre la prensa generalista y la prensa económica, en cuanto a áreas temáticas del periodismo jurídico.

- En último lugar mostrar el desarrollo y evolución de estas áreas durante treinta años de democracia (19812011), así como verificar que en los últimos años de análisis este tipo de información incrementa. Esto nos mostrará cuál es la situación del periodismo jurídico en estos momentos.

En cuanto a las hipótesis, nos planteamos las siguientes:

1. El análisis de la información jurídica en cada una de sus temáticas: sucesos, tribunales, rosa, economía y política; en los periódicos, tanto especializados como generalistas. Creemos que se constituyen tres grandes áreas de información jurídica en la prensa: Sociedad, Economía y Política.

2. Presumimos que la información jurídica en la prensa especializada (económica) tiene unas áreas temáticas de información jurídica completamente diferentes a las de la prensa generalista.

3. Suponemos que el periodismo jurídico está en auge, debido a la cantidad de información jurídica sobre casos de corrupción que ofrecen actualmente los medios. Por ello también suponemos que la información jurídicopolítica debe ser la que más sobresalga en este análisis.

\section{METODOLOGÍA}

Para la elaboración de esta investigación se empleará un análisis cualitativo y un análisis cuantitativo:

El análisis cualitativo centra su estudio en el análisis de contenido descriptivo, que como señalan los profesores Hernández, Fernández y Baptista (2007: 299), el investigador tiene como objetivo describir situaciones, eventos y hechos mediante la recolecta de datos.

El análisis cuantitativo es un tipo de análisis estadístico de los datos que en un principio se han recolectado. En él se realizarán análisis estadísticos con una variable, en este caso la sección donde aparece la información analizada, para luego analizar la relación de esta variable entre los diferentes diarios. Este tipo de análisis estadístico se efectuará con gráficos explicativos, donde se mostrarán datos sobre el desarrollo de la investigación.

Se han escogido tres medios de comunicación impresos: dos generalistas: Abc y El País; y otro de información especializada, en este caso un periódico económico: Cinco Días. El motivo es que se quiere comparar dos 
generalistas de gran tirada para ver diferencias y semejanzas en las secciones, y un económico, puesto que en estos periódicos especializados se concentra gran cantidad de información jurídica.

El análisis de los tres diarios se realiza desde 1981 hasta 2011. Viendo así los primeros años de democracia en España y su evolución hasta la actualidad con respecto a la información jurídica.

La razón por la cual el análisis empieza en 1981 es porque es el primer año en que coincide la existencia de los tres diarios señalados.

La muestra consiste en un día cualquiera de un mes al azar de cada año que entra dentro del análisis: por ejemplo, el 3 de marzo de 1981, el 17 de junio de 1982, etc; lo que viene a resumirse en la elección de un periódico por año, de esta manera se consigue una muestra pequeña de cada periódico, para hacer una comparativa de los tres diarios.

En total se han analizado un total de 508 informaciones jurídicas: 278 de Abc, 157 de El País y 73 de Cinco Días.

Este método de investigación se adapta a Berelson, que muestra el análisis de contenido como "una técnica de investigación para la descripción objetiva, sistemática y cuantitativa del contenido manifiesto de la comunicación" (Berelson, 1984: 18), lo que sirve de base metodológica para este estudio.

Sin embargo, se considera esta técnica en sentido más amplio, como señalan Piñuel y Gaitán al definirlo como "conjunto de procedimientos interpretativos y de técnicas de comprobación y verificación de hipótesis aplicadas a productos comunicativos (mensajes, textos o discursos) o a interacciones comunicativas que, previamente registradas, constituyen un documento, con el objeto de extraer y procesar datos relevantes" (Piñuel y Gaitán, 1995: 511).

Esta investigación implica la admisión tanto del análisis cualitativo como del análisis del contenido latente de las comunicaciones, como se recogen en los elementos a considerar en la ficha de análisis:

- Fecha de publicación.Indica la fecha en la que se publica la información con el orden siguiente: día/mes/año.

- Periódico.Señala cuál es el medio analizado. En este caso puede ser Abc, El País y Cinco Días.

- Titular.Se refiere al titular de la información jurídica seleccionado para analizar.

- Sección. Localiza la sección donde aparece la información jurídica.

\section{LAS ÁREAS DEL PERIODISMO JURÍDICO}

Antes de entrar en materia en las clases de periodismo jurídico que existen es importante saber cuáles son las áreas de información que aparecen en los medios de comunicación generalistas y que a su vez son información especializada:

1. Información política.

2. Información internacional.

3. Información económica.

4. Información cultural.

5. Información sobre sociedad.

6. Información sobre deportes.

7. Otras informaciones que no suelen ser fijas pero que a veces aparecen son las que tratan sobre ciencia, salud y tecnología. 
8. Gente, Televisión y Radio, Comunicación.

En prensa, la información política se sitúa en la sección Nacional/España e Internacional. La de sociedad recoge diferentes temáticas que no tienen suficiente independencia para tener su sección fija, estas son: Sanidad, Ecología, Religión, Sucesos, Tribunales, información Rosa, etc.

En las últimas páginas del periódico siempre aparecen secciones que llevan por nombre Gente, Televisión y Radio, o Comunicación, dependiendo del periódico. Estas áreas son las que hablan de todo lo relacionado con los medios de comunicación.

Por su parte, el periodismo jurídico abarca cualquiera de las temáticas anteriormente señaladas, pero como materias específicas predominan:

\section{Sucesos}

2. Tribunales

3. Económico

4. Político

\section{Famosos, información Rosa}

6. Otras (Salud, Ciencia, Tecnología, Deportes)

En la sección Otras se incluyen varios bloques temáticos del periodismo porque se considera que cada medio de comunicación divide los temas según sus intereses.

La información jurídica va ligada asiduamente a alguna de estas materias específicas. A veces se da el caso de una información donde se mezclan dos áreas, un ejemplo sería la boda de un futbolista o el juicio de un político. En el primer caso la información podría aparecer en Deportes o en Sociedad, y en el segundo caso, en Política o Tribunales, que en ese supuesto también sería Sociedad, puesto que Tribunales se sitúa dentro.

Es conveniente saber en qué sección se concentra la mayor parte de la información jurídica. A continuación se muestra una tabla y un gráfico que representa el número de veces (frecuencia) que aparece información jurídica en cada sección del diario Abc, desde el año 1981 hasta 2011.

El apartado denominado Otras se refiere a las siguientes secciones: Deportes, Madrid, portada Madrid, Sumario, Sumario Madrid y Sin Sección. En cuanto a Madrid es toda la información local de la ciudad, que lleva sumario y portada independiente de la general. Las consideradas sin sección son aquellas primeras páginas del periódico donde aparece información jurídica y no se señala un nombre.

\begin{tabular}{|l|l|l|}
\hline Secciones de Abc (1981-2011) & Frecuencia & Porcentajes (\%) \\
\hline Secciones & 110 & 39,57 \\
\hline España/Nacional & 14 & 5,04 \\
\hline Internacional & 26 & 9,35 \\
\hline Economia & 19 & 6,83 \\
\hline Sociedad & 10 & 3,6 \\
\hline Sucesos & 11 & 3,96 \\
\hline Portada & 9 & 3,24 \\
\hline Comunicación/Gente & 15 & 5,4 \\
\hline Actualidad gráfica & 5 & 1,8 \\
\hline Opinión & 59 & 21,22 \\
\hline Otras & 5 & 100 \\
\hline Total: 278 informaciones juridicas & \\
\hline
\end{tabular}

Secciones de Abc (1981-2011).

Fuente: Elaboración propia. 


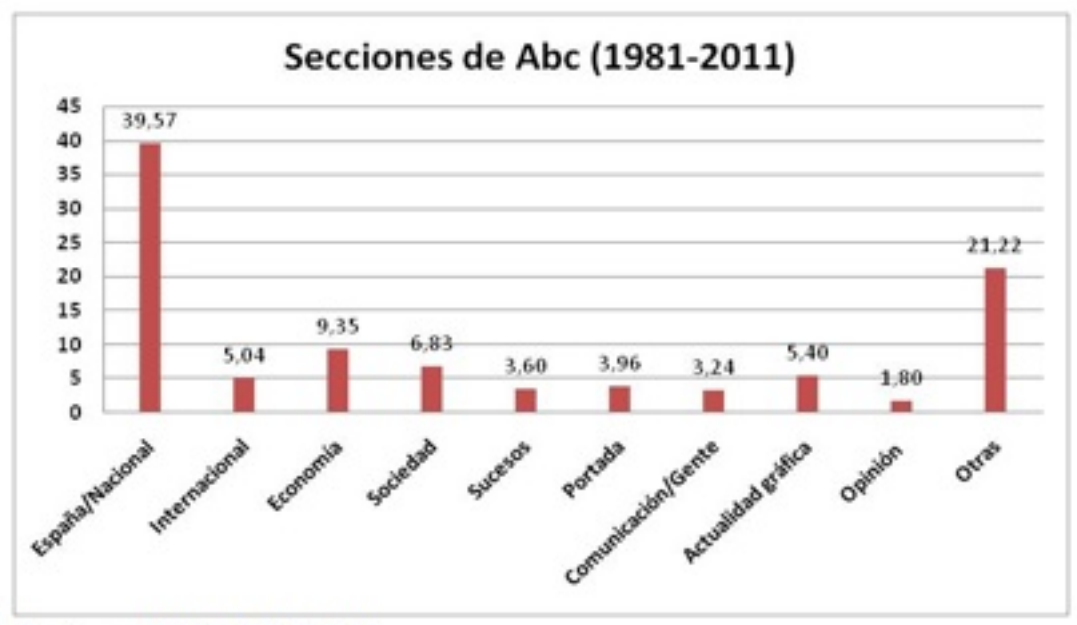

Secciones de Abc (1981-2011).

Fuente: Elaboración propia.

Teniendo en cuenta las materias específicas del periodismo jurídico, de un total de 278 informaciones jurídicas analizadas en $A b c$ desde 1981 hasta 2011, casi un 40 por ciento se concentran en la sección España/Nacional (Política), un cinco por ciento en Internacional (Política), un nueve por ciento en Economía, casi un siete por ciento en Sociedad, casi un cuatro por ciento en Portada y Sucesos (hasta 1998 que la sección desaparece y en 1999 sus contenidos se incluyen en Sociedad).

La Actualidad Gráfica se refiere a las primeras páginas del periódico que son fotonoticias y simbolizan el cinco por ciento.

Las secciones relacionadas con los medios de comunicación como Comunicación/Gente representan un tres por ciento, la sección de Opinión casi un dos por ciento, y Otras un 21 por ciento, donde se incluyen todas secciones nombradas anteriormente entre las que se encuentra Deportes.

La razón por la que Sucesos se distingue de Sociedad es porque fue una sección independiente hasta 1998, por eso se ha incluido en el gráfico, ya que a partir de 1999 sus contenidos pasan a formar parte de la sección Sociedad.

\begin{tabular}{|l|l|l|}
\hline Secciones de El Pais (1981-2011) \\
\hline Secciones & Frecuencia & Porcentajes (\%) \\
\hline Nacional/España & 59 & 37,58 \\
\hline Internacional & 13 & 8,28 \\
\hline Economia & 10 & 6,37 \\
\hline Sociedad & 31 & 19,75 \\
\hline Portada & 11 & 7,01 \\
\hline Otras & 33 & 21,02 \\
\hline Total: 157 informaciones juridicas & 100 \\
\hline
\end{tabular}

Secciones de El Pais (1981-2011).

Fuente: Elaboración propia. 


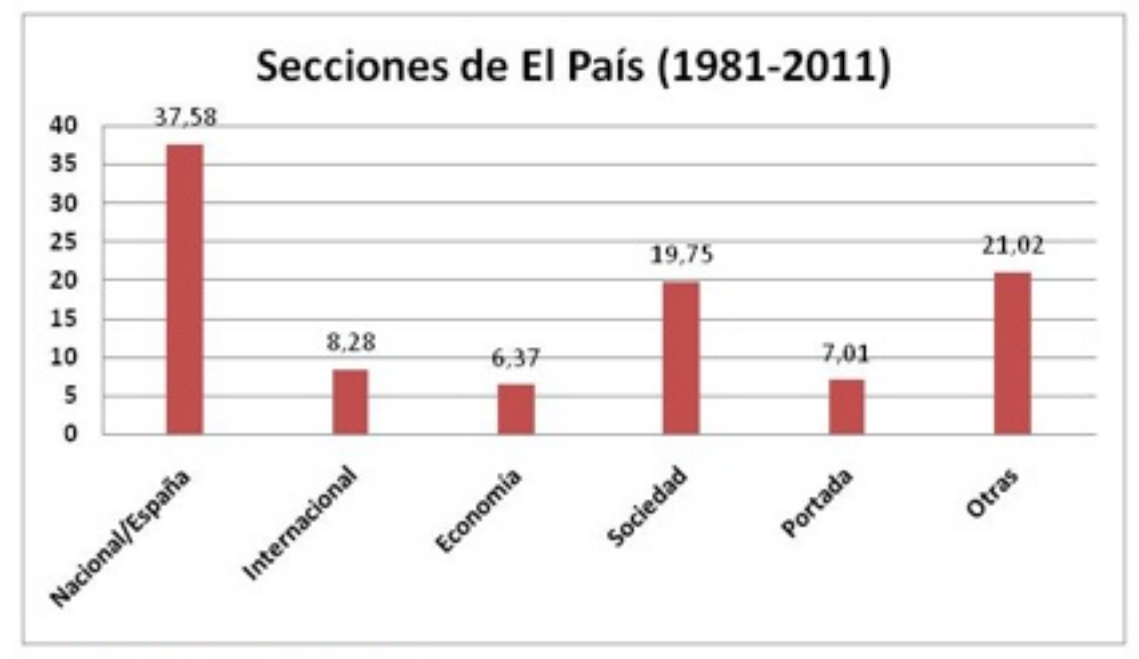

Secciones de El Pais (1981-2011).

Fuente: Elaboración propia.

Al igual que en $A b c$ en el diario El País también predomina la información jurídica en las secciones de España/Nacional (Política) con casi un 38 por ciento.

De las 157 informaciones jurídicas analizadas, un 20 por ciento representan la sección de Sociedad, donde se incluyen los Sucesos y Tribunales entre otras subsecciones, un ocho por ciento Internacional, un seis por ciento Economía, un siete por ciento Portadas y un 21 por ciento Otras.

En El País, Otras hace referencia a portadas de la Comunidad Valenciana, Comunidad Valenciana, Madrid, Televisión/Radio, Cultura y Deportes.

Opinión no se ha incluido porque durante el análisis no se han encontrado información jurídica en esa sección.

La diferencia entre ambos periódicos generalistas no dista mucho, ya que ambos incluyen las mismas secciones principales.

En el periodismo jurídico de la prensa generalista destacan por tanto tres áreas grandes de información jurídica: Política, Economía y Sociedad.

Se entiende que el área Política centra sus informaciones jurídicas en las secciones España/Nacional e Internacional. En los últimos años ha habido una tendencia al alza en la información jurídica que se centra estas secciones aunque más en la primera sección. Esto es debido al crecimiento de los casos de corrupción, donde parece ser que la política se esté judicializando, puesto que todos los días salen noticias nuevas sobre alguna trama y la actualidad política gira en torno a este tipo de noticias.

El área Sociedad tiene temáticas diferentes pero principalmente como información jurídica incluye Sucesos, Tribunales e información sobre gente famosa perteneciente al periodismo Rosa.

En Economía aparece también información jurídica y es la única área cuya sección se denomina igual.

Secciones como Deportes o Comunicación, son menos relevantes y algunas veces aparece esta clase de información pero en menor medida.

Por este motivo, la información jurídica se mueve en diferentes áreas informativas y no tiene un área fija, y a la vez, se puede encontrar en cualquier sección de las citadas anteriormente.

\subsection{Sociedad}

La sección Sociedad es una sección común en todos los periódicos generalistas, además de ser una de las secciones más amplias en cuanto a contenidos se refiere, puesto que en ella se encuentran noticias relacionadas con temas sociales, religión, educación, sucesos, tribunales, noticias sobre personas famosas, etc. 
Las materias que se incluyen en esta sección tienen unos criterios de elección según Gallego (2003: 97):

a) Asuntos de actualidad

b) Asuntos atemporales, pero que tienen interés social

c) Temas de investigación que se emiten o publican con carácter especial

Continúa apuntando Gallego (2003a: 97) que estas áreas de Sociedad surgen de los siguientes ámbitos temáticos:

a) Sucesos, catástrofes, accidentes y hechos fortuitos;

b) Problemas sociales de interés general;

c) Tendencias y cambios perceptibles en la sociedad;

d) Vida cotidiana de grupos e individuos.

La sección Sociedad carece de rigor respecto a otras secciones del periódico como pueden ser Economía, Deportes o Política, pues a lo largo de su existencia Sociedad ha estado en constante cambio y modificaciones, por lo que los propios periódicos no han sabido estructurar sus contenidos como lo han hecho con otras áreas.

El primer periódico que incluye la sección Sociedad es El País, que nace en 1976. Pero el cambio desde aquel entonces hasta ahora en la actualidad ha sido grande, puesto que las informaciones que incluían no tienen nada que ver con las de ahora, ya que por los años se han ido incorporando nuevos contenidos y nuevos enfoques.

$A b c$ cuenta con un número de páginas mayor que otros periódicos, por ello puede permitirse además de contener la sección Sociedad el incluir secciones independientes como Religión, Sanidad, Educación, etc. El resto de diarios las han suprimido e incluido en Sociedad.

A parte de una falta de concreción, existe cierta diversidad de títulos y cambios de nombre en esta sección. Además, el nacimiento de esta área nace a raíz de una reestructuración tecnológica que sufre la prensa diaria, a mediados de los años setenta. En esta época desaparecen periódicos y aparecen nuevos, y con la nueva situación político-económica también son diferentes las necesidades informativas, por este motivo se moderniza no solo el país sino también las redacciones de los periódicos, que para poder sobrevivir a este cambio tienen que reorganizar sus plantillas y sus contenidos.

Con este cambio, secciones fijas como Tribunales y Sucesos son insuficientes para continuar publicándose por solitario, pues no pueden seguir ofreciendo información diaria adecuada a la nueva situación. Estas áreas ya no pueden considerarse independientes y empiezan a formar parte de un área en común donde los contenidos ya no tienen un espacio propio pero se profundiza más en ellos.

Teniendo en cuenta que nos encontramos ante una sección cuyos temas son de lo más variados, se puede concretar que la información jurídica en el área de Sociedad se compone por tres bloques temáticos principalmente: Sucesos, Tribunales y Rosa.

El periodismo de sucesos hace referencia a hechos peculiares, extraños y a veces de gran impacto, los cuales aparecen en la vida cotidiana de las personas anónimas o no famosas, provocando víctimas. Un suceso pueden ser tragedias como la pérdida de un hijo, un incendio, el robo en una casa o comercio, etc.

Aunque se muestran como temáticas diferentes, Sucesos y Tribunales siempre han estado relacionados. Como dice Quesada (2007: 40) la mayoría de hechos que se dan en esta clase de periodismo, actualmente tienen que ver también con tribunales, puesto que ellos deben intervenir para aplicar las leyes.

Javier Ronda y José María Calero (2000: 23) piensan que el periodismo de sucesos es una categoría del periodismo de tribunales, siendo este último el concepto más genérico. 
María José Pou (2013: 133) lo define como un periodismo que abarca hechos cotidianos.

Los tres autores coinciden en que cuando el periodismo de sucesos también narra hechos delictivos, aquí intervienen los tribunales para aplicar las leyes, dando lugar al trabajo de los periodistas de tribunales, que aparecen para cubrir los juicios e informar de los hechos.

Este tipo de periodismo puede abarcar una sentencia, una denuncia, la declaración de un testigo, la condena de un juez, etcétera; de ahí que el periodista que trabaja en esta clase de información tenga que dedicarle un seguimiento especial, ya que a veces los procesos judiciales son largos.

Dentro de esta clase de información aparecen subáreas, lo que complica el trabajo del periodista. Con ello nos referimos a que dependiendo de la información que se cubra pertenecerá a una clase de proceso u otro. Javier Ronda (2002-2003: 187) lo explica así: "este periodismo está relacionado con la actividad que emana de las diferentes sedes judiciales. Desde un juzgado de menores, hasta el Tribunal Constitucional, desde el Supremo hasta un juzgado de paz. Recursos, comparecencias, autos y, por supuesto, las sentencias y los juicios".

Mientras el periodismo de sucesos se encarga de informar sobre el hecho en el momento en que se está produciendo, el periodismo de tribunales hace el seguimiento del caso en los tribunales, hasta el momento en que el juez dicta la resolución, momento de mayor interés informativo en el cual se cierra el caso definitivamente.

La información Rosa es otra temática principal dentro del área Sociedad. Aunque esta clase de periodismo no es muy popular en la prensa, ya que existen revistas especializadas, la sección Sociedad siempre ha contado con ella, según Maite Mercado (1999) "la información del corazón se constituiría como un subárea dentro de este bloque temático denominado Sociedad; no en vano en muchas ocasiones a este tipo de información se la conoce también como noticias de sociedad".

En relación al mundo jurídico, la información Rosa siempre suele incluir los mismos temas: divorcios, custodias, demandas y querellas cuando se atenta contra el derecho al honor y a la imagen.

Concretamente, la información jurídica en la sección de Sociedad acapara tres clases de contenidos principales: Sucesos, Tribunales y Rosa. Esto solo pasa en la prensa generalista ya que en la económica prescinde de esta sección, puesto que su información y temas son de otra índole.

\subsubsection{Desarrollo y evolución de la información jurídica en la sección de Sociedad (1981-2011)}

Los sucesos fueron una de las informaciones que sufrieron cambios, pues en algunos periódicos tenían una sección independiente y con los años esta clase de noticias pasaron a incluirse en Sociedad. Un ejemplo es el periódico Abc cuya sección de Sucesos permaneció hasta 1998, y a partir de 1999 era Sociedad la sección que incluía este tipo de informaciones.

El País nunca ha tenido sección de Sucesos, pues desde su nacimiento en 1976 este tipo de información siempre se ha incluido en Sociedad. Esto puede ser porque El País ya nace en plena transición española, por lo que los contenidos se enfocan ya de diferente manera. En cambio, $A b c$, al igual que otros periódicos más veteranos, tuvo que ir modificándose con los nuevos cambios políticos y socio-económicos.

De esta evolución de los sucesos a la sección de Sociedad habla Montserrat Quesada (2010: 58) refiriéndose a una única sección donde van a parar todas aquellas noticias que no tienen espacio en el resto del periódico.

El periódico económico Cinco Días, por su misma especialidad, nunca ha publicado este tipo de noticias, por lo que no hay referencias.

La información de Tribunales, aunque ahora está muy presente en los medios de comunicación, empieza a tener auge a finales de los años 70 y principios de los 80 , con la etapa democrática.

La presencia de este tipo de información va relacionada con la ampliación del ámbito de actuación de juzgados y tribunales, por lo que ha despertado un mayor interés entre la ciudadanía (3). 
En $A b c$, los contenidos que hacen referencia a tribunales se incluyen en la sección Sociedad, esto es constante durante todo el análisis hasta 1995, donde el diario decide incluir en sus páginas la sección Tribunales, la cual solo aparece ese año, puesto que en 1996 desaparece y sus contenidos vuelven a formar parte de la sección Sociedad.

A diferencia de $A b c$, el periódico El País, nunca ha tenido sección de Tribunales pero esta sí que aparece dentro de Sociedad como una subsección, aunque esto no ha pasado siempre, ya que también se ha podido ver Tribunales dentro de Política con José Yoldi. Es muy propio de El País tener subsecciones dentro de Sociedad, como por ejemplo Sociedad (Tribunales), Sociedad (Sanidad), Sociedad (Comunicación), etc.

En el periódico económico Cinco Días, la sección Sociedad no existe, pero sí que existió una sección de Tribunales en el período de años que va desde 1995 hasta 2001, luego esta sección ya no se encuentra entre las páginas del diario, aunque sí que es cierto que actualmente existe una sección llamada Legislación. Esta no aparece a diario pero está presente. En ese período donde existía la sección de Tribunales, destacan los años 1997 y 1998, que son los de mayor publicación. Los temas principales en estos dos años de mayor auge son los relacionados con Mario Conde y el juez Moreiras, y el caso Sogecable.

En 2002 esta sección de Tribunales desaparece, y esta información se incluye en la sección Empresas principalmente. Actualmente, esta sección destaca por concentrar un número mayor de información jurídica.

Los órganos de la administración de justicia, las sentencias, condenas, juicios, etc., hacen referencia a los temas de información de tribunales que ofrecen los periódicos generalistas. El periodismo de sucesos y el de tribunales son diferentes pero ambos se hallan en la misma sección de Sociedad, por este motivo en ocasiones no se deja ver claramente donde acaba el trabajo del periodista de sucesos y donde empieza el del periodista de tribunales.

Existe una gran diferencia entre ambos tipos de periodismo y es que la temática de Sucesos apenas tiene movimiento dentro del periódico, pues es difícil que un asesinato o robo se vea en la sección España/Nacional. En cambio, la información de Tribunales está en constante movimiento, de ahí se entiende también que no tenga una sección fija, puesto que si hay un juicio sobre un político corrupto no lo veremos publicado en Sociedad sino en Política o Nacional.

En el periódico especializado la información de Tribunales también recoge esa temática, pero como se ha dicho anteriormente no se puede hablar de una sección de Sociedad, puesto que esta no existe.

En la sección de Sociedad también se incluye la información jurídica del periodismo Rosa, donde los temas que se tratan principalmente son: bodas, romances, divorcios, demandas y querellas de famosos cuando atentan al derecho a su imagen o a su honor, etc. Estas "noticias de sociedad" que hacen referencia al mundo de la prensa rosa o prensa del corazón tampoco tienen una sección específica en la prensa generalista. Es un tipo de información que se puede encontrar no solo en Sociedad sino en cualquier área, puesto que al tratarse de gente que forma parte del espectáculo, la televisión, la cultura o el deporte, dependerá de qué personaje sea y el motivo por el cual es noticia.

Este periodismo Rosa se diferencia de otras clases de periodismo por una serie de características, que tal y como establece Mar de Fontcuberta (1990: 61) son: predominio absoluto de los personajes; cultivo del interés humano frente al interés público de la prensa informativa; una actualidad que oscila entre los dos niveles: la exclusividad del último momento y la ahistoricidad de gran parte de sus contenidos; la novedad, la noticia excepcional, viene matizada por una dedicación importante a las informaciones cotidianas y rutinarias, que no suponen ninguna ruptura con la norma; utilización de lo verisímil frente a lo veraz; periodicidad en su aparición ante el público, con una frecuencia generalmente semanal.

En la siguiente tabla se detalla la frecuencia en la que la información jurídica aparece en la sección de Sociedad en los diarios Abc y El País, entre 1981 y 2011. 


\begin{tabular}{|c|c|c|}
\hline Años & Frecuencia (Información Juridica Sociedad) & \begin{tabular}{|l|} 
Porcentaje \\
$(\%)$
\end{tabular} \\
\hline 1981 & 0 & 0 \\
\hline 1982 & 0 & 0 \\
\hline 1983 & 0 & 0 \\
\hline 1984 & 1 & 3,45 \\
\hline 1985 & 0 & 0 \\
\hline 1986 & 1 & 3,45 \\
\hline 1987 & 1 & 3,45 \\
\hline 1988 & 0 & 0 \\
\hline 1989 & 0 & 0 \\
\hline 1990 & 2 & 6,9 \\
\hline 1991 & 3 & 10,34 \\
\hline 1992 & 0 & 0 \\
\hline 1993 & 2 & 6,9 \\
\hline 1994 & 2 & 6,9 \\
\hline 1995 & 1 & 3,45 \\
\hline 1996 & 0 & 0 \\
\hline 1997 & 2 & 6,9 \\
\hline 1998 & 2 & 6,9 \\
\hline 1999 & 5 & 17,24 \\
\hline 2000 & 0 & 0 \\
\hline 2001 & 1 & 3,45 \\
\hline 2002 & 2 & 6,9 \\
\hline 2003 & 0 & 0 \\
\hline 2004 & 1 & 3,45 \\
\hline 2005 & 1 & 3,45 \\
\hline 2006 & 2 & 6,9 \\
\hline 2007 & 0 & 0 \\
\hline 2008 & 0 & 0 \\
\hline 2009 & 0 & 0 \\
\hline 2010 & 0 & 0 \\
\hline 2011 & 0 & 0 \\
\hline \multicolumn{2}{|c|}{ Total: 29 informaciones juridicas } & 100 \\
\hline
\end{tabular}

La información jurídica en la sección de Sociedad en Abc (1981-2011).

Fuente: Elaboración propia.

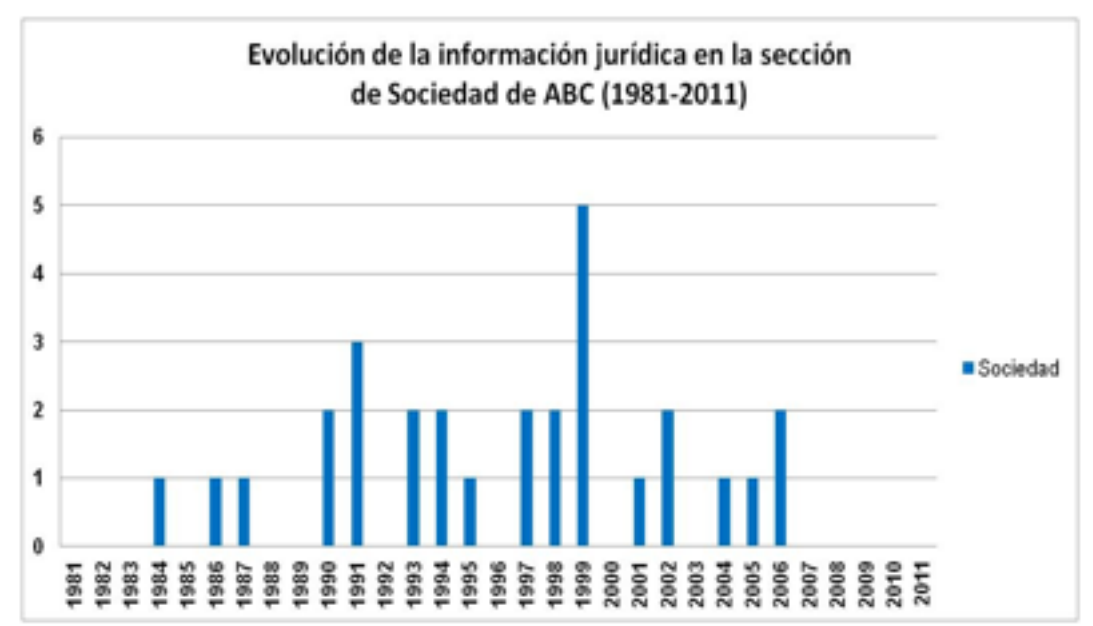

Evolución de la información jurídica en la sección de Sociedad en Abc (1981-2011).

Fuente: Elaboración propia.

La evolución de la información jurídica en la sección Sociedad en $A b c$ no es tan constante, es más, existen unos años donde no se publica, estos son de 1981 hasta 1983, 1985, 1988, 1989, 1992, 1996, 2000, 2003, y del 2007 hasta el 2011. Esto no significa que no se haya publicado información jurídica, sino que esta se ha publicado en otras secciones.

El año más destacado en número de informaciones jurídicas es 1999, esto sucede porque en 1998 desaparece la sección de Sucesos y toda esa información empieza a publicarse en Sociedad.

En cuanto al período que va desde 2007 hasta 2011, en estos últimos años la publicación de información 
jurídica se concentra mucho en la sección Nacional/España, como se verá en el punto 4.3.1.

El País tiene un total de treinta y una informaciones jurídicas en la sección Sociedad. Cabe recordar que este diario nunca tuvo sección de Sucesos, puesto que desde el nacimiento del periódico este tipo de informaciones se incluyeron en Sociedad hasta la actualidad.

\begin{tabular}{|c|c|c|}
\hline Años & Frecuencia (Información Juridica Sociedad) & $\begin{array}{l}\text { Porcentaje } \\
(\%)\end{array}$ \\
\hline 1981 & 0 & 0,00 \\
\hline 1982 & 1 & 3,23 \\
\hline 1983 & 0 & 0,00 \\
\hline 1984 & 1 & 3,23 \\
\hline 1985 & 1 & 3,23 \\
\hline 1986 & 0 & 0,00 \\
\hline 1987 & 4 & 12,90 \\
\hline 1988 & 2 & 6,45 \\
\hline 1989 & 4 & 12,90 \\
\hline 1990 & 3 & 9,68 \\
\hline 1991 & 0 & 0,00 \\
\hline 1992 & 1 & 3,23 \\
\hline 1993 & 2 & 6,45 \\
\hline 1994 & 0 & 0,00 \\
\hline 1995 & 0 & 0,00 \\
\hline 1996 & 0 & 0,00 \\
\hline 1997 & 2 & 6,45 \\
\hline 1998 & 1 & 3,23 \\
\hline 1999 & 1 & 3,23 \\
\hline 2000 & 2 & 6,45 \\
\hline 2001 & 0 & 0,00 \\
\hline 2002 & 0 & 0,00 \\
\hline 2003 & 0 & 0,00 \\
\hline 2004 & 0 & 0,00 \\
\hline 2005 & 0 & 0,00 \\
\hline 2006 & 1 & 3,23 \\
\hline 2007 & 1 & 3,23 \\
\hline 2008 & 0 & 0,00 \\
\hline 2009 & 0 & 0,00 \\
\hline 2010 & 4 & 12,90 \\
\hline 2011 & 0 & 0,00 \\
\hline \multicolumn{2}{|c|}{ Total: 31 informaciones juridicas } & 100 \\
\hline
\end{tabular}

La información juridica en la sección de Sociedad en El Pais (1981-2011).

Fuente: Elaboración propia.

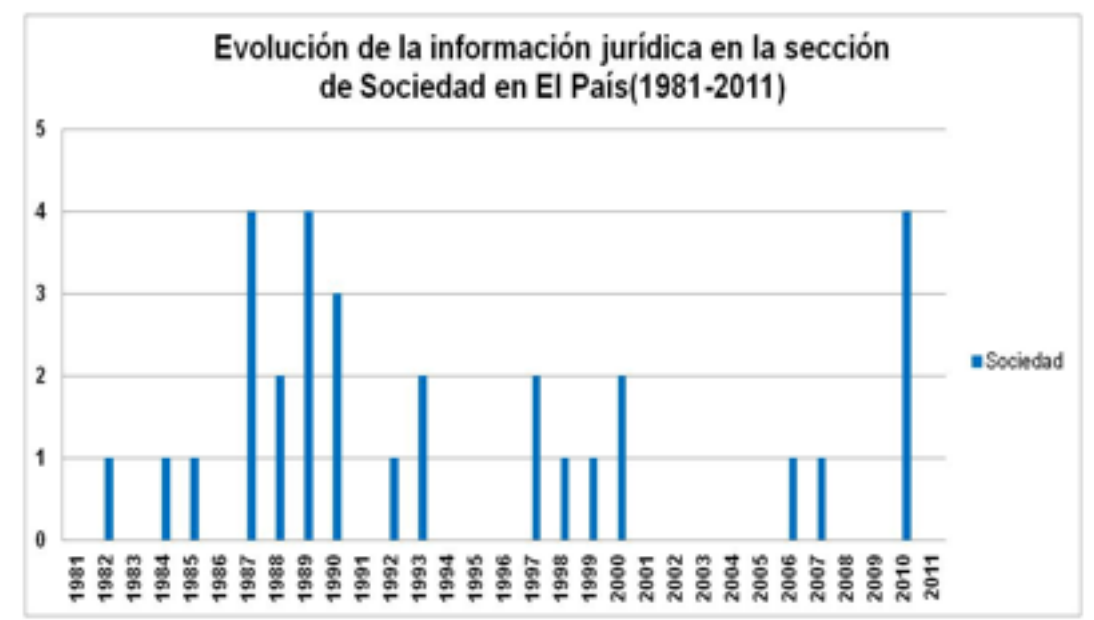

Evolución de la información jurídica en la sección de Sociedad en El Pais (1981-2011).

Fuente: Elaboración propia.

En los primeros años de análisis la evolución de esta información en la sección Sociedad es firme, pero a partir del año 1994 empieza a tener altibajos, existen períodos cortos de años donde se publica más y otro donde no se publica, así que no se puede afirmar que haya una regularidad. Los años que destacan por el número de publicaciones son 1987, 1989 y 2010, tal y como aparece coloreado en la tabla y representado en el gráfico. En estos años la información que destaca es la de tribunales. 
Los períodos de años seguidos donde no se publica información jurídica en la sección Sociedad, destacan de 1994 a 1996 y de 2001 a 2005. En ambos períodos la información jurídica se publica en la sección España.

\subsection{Economía}

La información especializada que trata sobre el mundo de las empresas, las finanzas, la bolsa, los negocios, los mercados, etc. es información económica, y su finalidad es informar facilitando la comprensión a los lectores sobre todas estas áreas que engloban el marco económico en la sociedad actual en la que vivimos.

Cuando este tipo de información sobre empresas, negocios, mercados, etc., se entremezcla con legislaciones, demandas, querellas, sentencias, leyes, resoluciones, normas, juicios, etc., estamos ante información jurídicoeconómica.

La prensa económica profundiza más que la generalista en cuanto a materias jurídicas se refiere, pues proporciona noticias sobre despachos de abogados, textos legales, etc. Además, los diarios económicos cuentan con un espacio exclusivo para hablar de información jurídica, tales como secciones, suplementos o revistas, donde intervienen colaboradores que pertenecen al mundo jurídico, como abogados, procuradores, etc., proporcionando así una doble visión de la información jurídica: la del periodista y la de la persona especializada. Por el contrario, la prensa generalista no tiene un sitio donde ubicar esta información, sino que esta se mueve en diferentes áreas temáticas.

\subsubsection{Desarrollo y evolución de la información jurídico-económica en la prensa generalista (1981-2011)}

En los periódicos generalistas la sección economía siempre ha estado presente. El tener una sección fija no siempre es habitual en todas las áreas, pero en esta sí. A continuación vamos a ver la evolución que ha tenido esta sección en los diarios Abc y El País. 


\begin{tabular}{|c|c|c|}
\hline Años & Frecuencia (Información Juridica Economia) & $\begin{array}{l}\text { Porcentaje } \\
(\%)\end{array}$ \\
\hline 1981 & 1 & 3,85 \\
\hline 1982 & 0 & 0,00 \\
\hline 1983 & 1 & 3,85 \\
\hline 1984 & 1 & 3,85 \\
\hline 1985 & 1 & 3,85 \\
\hline 1986 & 1 & 3,85 \\
\hline 1987 & 0 & 0,00 \\
\hline 1988 & 0 & 0,00 \\
\hline 1989 & 1 & 3,85 \\
\hline 1990 & 0 & 0,00 \\
\hline 1991 & 1 & 3,85 \\
\hline 1992 & 1 & 3,85 \\
\hline 1993 & 3 & 11,54 \\
\hline 1994 & 0 & 0,00 \\
\hline 1995 & 1 & 3,85 \\
\hline 1996 & 1 & 3,85 \\
\hline 1997 & 2 & 7,69 \\
\hline 1998 & 0 & 0,00 \\
\hline 1999 & 1 & 3,85 \\
\hline 2000 & 0 & 0,00 \\
\hline 2001 & 2 & 7,69 \\
\hline 2002 & 0 & 0,00 \\
\hline 2003 & 1 & 3,85 \\
\hline 2004 & 3 & 11,54 \\
\hline 2005 & 0 & 0,00 \\
\hline 2006 & 2 & 7,69 \\
\hline 2007 & 1 & 3,85 \\
\hline 2008 & 0 & 0,00 \\
\hline 2009 & 1 & 3,85 \\
\hline 2010 & 0 & 0,00 \\
\hline 2011 & 0 & 0,00 \\
\hline \multicolumn{2}{|c|}{ Total: 26 informaciones juridicas } & 100 \\
\hline
\end{tabular}

La información jurídica en la sección de Economia en Abc (1981-2011).

Fuente: Elaboración propia.

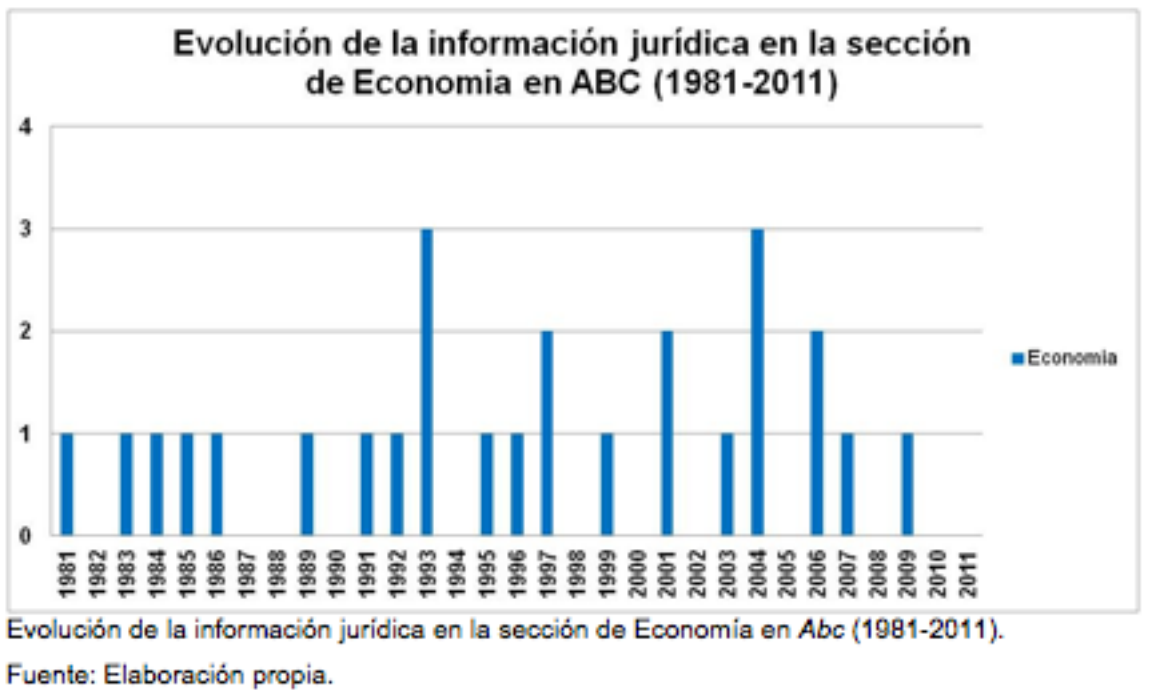

La publicación de información jurídica en la sección Economía no es tan constante, pero aún así, en más de la mitad de los años se publica información. Destacan 1993 y 2004 por tener más informaciones publicadas en la sección de Economía.

Igual que pasaba en la sección Sociedad, en la sección Economía también existen años donde no aparece información jurídica.

Esto no significa que no se publique información jurídica, sino que ésta se concentra principalmente en la sección de Nacional durante esos años. 
Concretamente en esta muestra de $A b c$ existen 26 informaciones jurídicas que han aparecido en la sección Sociedad entre 1981 y 2011.

Entre estos años, y tal y como aparece coloreado en la tabla de datos, en 1993 y 2004 se publican más informaciones que en otros años, en concreto tres, puesto que en el resto de años nos encontramos la mayoría de las veces con una información.

La sección Economía también forma parte del periódico El País, pero a diferencia de Abc la publicación de información jurídica en esta sección no ha sido tan constante.

Existen períodos largos de años donde no se publica información jurídica en la sección Economía, estos períodos son de 1985 hasta 1991, de 1995 hasta 1998, de 2000 hasta 2007, y 2009 y 2010 . En todos ellos la información jurídica aparece en secciones como Sociedad, España e Internacional

4.2.2. Desarrollo y evolución de la información jurídica en la prensa económica a través de Cinco Días (19812011)

En Cinco Días, al tratarse de un periódico económico no es aconsejable seleccionar como representación solo la sección de Economía, así que en el siguiente gráfico, aparece la evolución de la información jurídica en todas las secciones del diario.

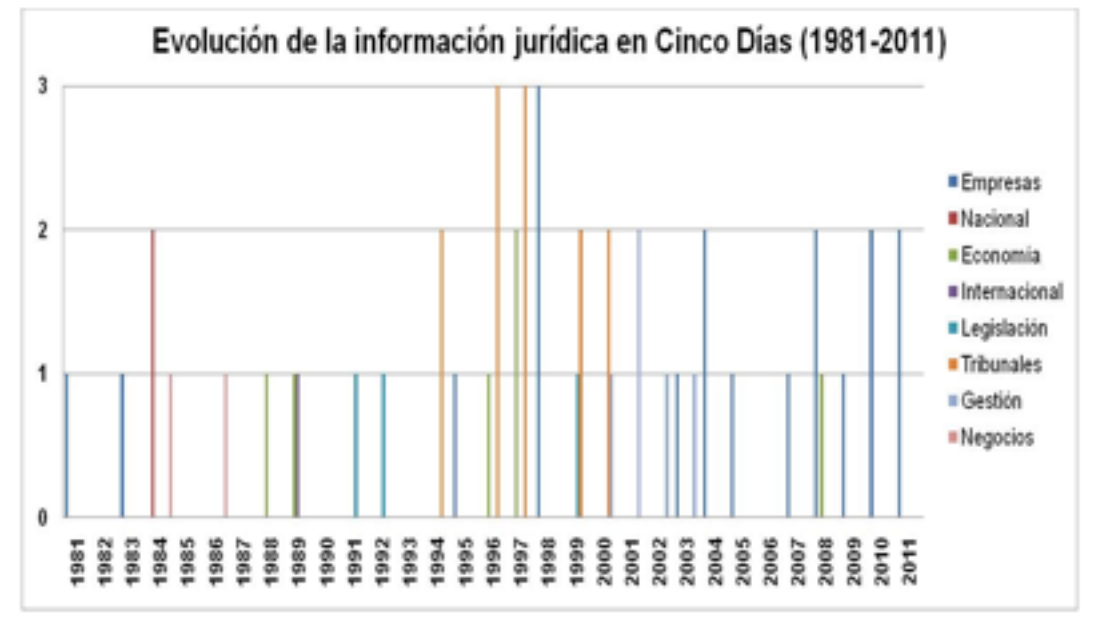

Evolución de la información jurídica en Cinco Dias (1981-2011).

Fuente: Elaboración propia.

La información jurídica en la sección Economía de Cinco Días apenas tiene relevancia, sin embargo, en los últimos años se ha notado un incremento de este tipo de información en la sección Empresas, puesto que desde 2002 hasta la actualidad es la información que más destaca. Al igual que está pasando en la prensa generalista donde la información jurídica se concentra principalmente en las secciones de Nacional y España.

Esto significa que en la prensa económica la información jurídica relacionada con el mundo empresarial es la que está en auge, mientras que en la prensa generalista la que más predomina es la información jurídica relacionada con la política.

En cuanto al resto de secciones en el periódico económico existen períodos cortos de publicación de información jurídica pero apenas han sido destacables, puesto que la sección que más ha publicado ha sido Empresas, como se ha comentado.

\subsection{Política}

La información política es la rama del periodismo especializado que trata la temática que rodea al mundo de la política, desde la nueva ley que ha adoptado el Gobierno hasta lo que opina la oposición de esta nueva ley, las campañas electorales, todas las decisiones que se toman concernientes a la sociedad, al bienestar de los ciudadanos y a la mejora del funcionamiento del sistema en el que convivimos. 
La información política, junto a la económica, es un pilar fundamental en el periodismo, pues en todos los estados el modelo político se complementa con el modelo económico. Este tipo de información influye mucho en la sociedad, pues en ocasiones, algunas decisiones políticas movilizan a las personas para manifestarse cuando no están de acuerdo con lo decidido.

Esta influencia que los medios ejercen en la sociedad mediante la información, hace que se denomine a la prensa el Cuarto Poder.

Cuando en este tipo de información se tocan temas relacionados con sentencias, juicios, demandas, querellas, etc., estamos hablando de información jurídico-política.

\subsubsection{Desarrollo y evolución de la información jurídico-política en la prensa generalista (1981-2011)}

Analizando la prensa generalista nos damos cuenta que no existe una sección fija para la información política, sino que esta se concentra mayormente en las áreas de España/Nacional e Internacional.

Cabe destacar que la sección Nacional de $A b c$ se llama así hasta el año 2006, puesto que en 2007 pasa a denominarse España. Por este motivo tanto en los datos que aparecen en la tabla como en el gráfico, se ha contabilizado la información jurídica de manera conjunta, puesto que son la misma sección solo que con el tiempo se ha cambiado el nombre.

\begin{tabular}{|c|c|c|}
\hline Años & $\begin{array}{l}\text { Frecuencia (Información Juridica } \\
\text { España/Nacional//nternacional) }\end{array}$ & $\begin{array}{l}\text { Porcentaje } \\
(\%)\end{array}$ \\
\hline 1981 & 2 & 1,61 \\
\hline 1982 & 2 & 1,61 \\
\hline 1983 & 0 & 0,00 \\
\hline 1984 & 3 & 2,42 \\
\hline 1985 & 1 & 0,81 \\
\hline 1986 & 1 & 0,81 \\
\hline 1987 & 5 & 4,03 \\
\hline 1988 & 4 & 3,23 \\
\hline 1989 & 6 & 4,84 \\
\hline 1990 & 6 & 4,84 \\
\hline 1991 & 3 & 2,42 \\
\hline 1992 & 2 & 1,61 \\
\hline 1993 & 4 & 3,23 \\
\hline 1994 & 2 & 1,61 \\
\hline 1995 & 4 & 3,23 \\
\hline 1996 & 7 & 5,65 \\
\hline 1997 & 5 & 4,03 \\
\hline 1998 & 9 & 7,26 \\
\hline 1999 & 3 & 2,42 \\
\hline 2000 & 3 & 2,42 \\
\hline 2001 & 2 & 1,61 \\
\hline 2002 & 6 & 4,84 \\
\hline 2003 & 3 & 2,42 \\
\hline 2004 & 3 & 2,42 \\
\hline 2005 & 4 & 3,23 \\
\hline 2006 & 3 & 2,42 \\
\hline 2007 & 6 & 4,84 \\
\hline 2008 & 7 & 5,65 \\
\hline 2009 & 6 & 4,84 \\
\hline 2010 & 4 & 3,23 \\
\hline 2011 & 7 & 5,65 \\
\hline \multicolumn{2}{|c|}{ Total: 124 informaciones juridicas } & 100 \\
\hline
\end{tabular}

La información jurídico-política en las secciones de España/Nacional e Internacional en $A b c$ (1981-2011).

Fuente: Elaboración propia. 


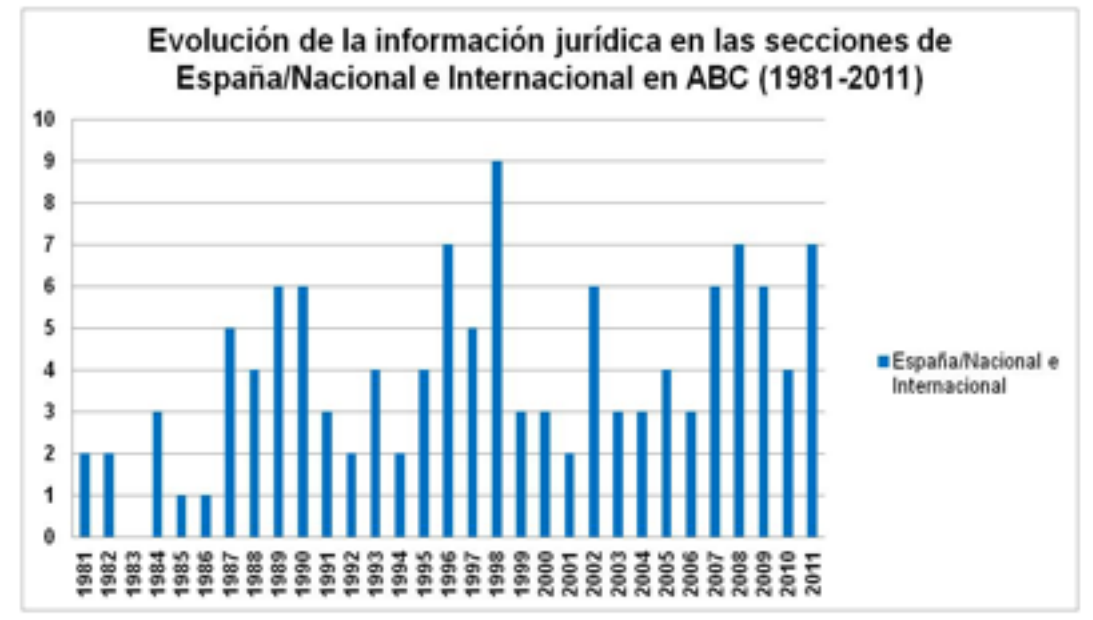

Evolución de la información jurídico-politica en las secciones de España/Nacional e Internacional en $A b c$ (1981-2011).

Fuente: Elaboración propia.

Menos en el año 1983, en el resto de años se ha publicado. Destaca 1998 por tener más número de informaciones jurídicas publicadas en las secciones de España/Nacional e Internacional. Entre estas informaciones el tema del que más se informa es sobre el "caso Marey".

La información jurídico-política ofrece una evolución constante, con algunos altibajos referentes a los días en los que se publica más o menos, pero no hay que olvidar que se trata de información jurídica y que puede aparecer en cualquier sección del periódico, lo que quiere decir que si un año tiene poco publicado en esa sección puede que se haya publicado más en otra u otras secciones. Por ello, al principio de esta investigación se muestra el número de informaciones jurídicas totales publicadas por años.

En el periódico El País, la sección Nacional pasó a llamarse España a partir de 1984, según el análisis.

A principios de la década de los ochenta, existía como una especie de subsección dentro de Nacional, la cual se llamaba Política, pero siempre perteneció a Nacional. 


\begin{tabular}{|c|c|c|}
\hline Años & $\begin{array}{l}\text { Frecuencia (Información Juridica } \\
\text { España/Nacional//nternacional) }\end{array}$ & $\begin{array}{l}\text { Porcentaje } \\
(\%)\end{array}$ \\
\hline 1981 & 2 & 2,78 \\
\hline 1982 & 0 & 0,00 \\
\hline 1983 & 0 & 0,00 \\
\hline 1984 & 1 & 1,39 \\
\hline 1985 & 3 & 4,17 \\
\hline 1986 & 1 & 1,39 \\
\hline 1987 & 2 & 2,78 \\
\hline 1988 & 1 & 1,39 \\
\hline 1989 & 3 & 4,17 \\
\hline 1990 & 0 & 0,00 \\
\hline 1991 & 2 & 2,78 \\
\hline 1992 & 3 & 4,17 \\
\hline 1993 & 1 & 1,39 \\
\hline 1994 & 5 & 6,94 \\
\hline 1995 & 3 & 4,17 \\
\hline 1996 & 8 & 11,11 \\
\hline 1997 & 0 & 0,00 \\
\hline 1998 & 0 & 0,00 \\
\hline 1999 & 4 & 5,56 \\
\hline 2000 & 2 & 2,78 \\
\hline 2001 & 2 & 2,78 \\
\hline 2002 & 2 & 2,78 \\
\hline 2003 & 8 & 11,11 \\
\hline 2004 & 2 & 2,78 \\
\hline 2005 & 4 & 5,56 \\
\hline 2006 & 2 & 2,78 \\
\hline 2007 & 4 & 5,56 \\
\hline 2008 & 0 & 0,00 \\
\hline 2009 & 4 & 5,56 \\
\hline 2010 & 2 & 2,78 \\
\hline 2011 & 1 & 1,39 \\
\hline \multicolumn{2}{|c|}{ Total: 72 informaciones juridicas } & 100 \\
\hline
\end{tabular}

La información jurídico-politica en las secciones de España/Nacional e Internacional en El Pais (1981-2011).

Fuente: Elaboración propia.

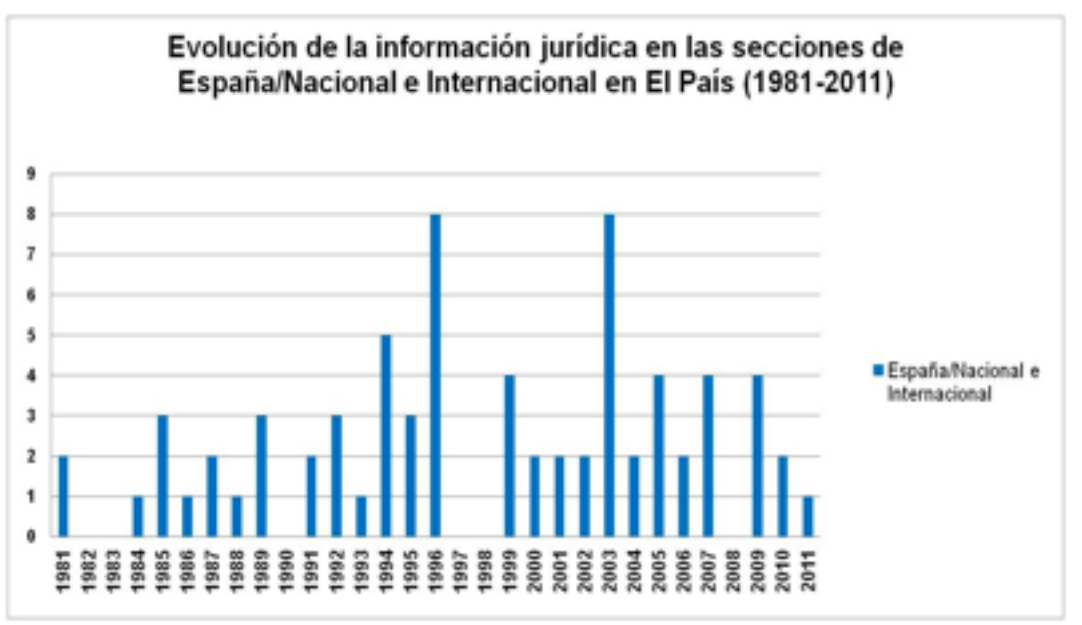

Evolución de la información juridico-politica en las secciones de España/Nacional e Internacional en El Pais (1981-2011).

Fuente: Elaboración propia.

Existen seis años concretamente donde no se publica información jurídica en estas secciones: 1982, 1983, 1990, 1997, 1998 y 2008. Al igual que en $A b c$, esto sucede porque la información jurídica en esos años se ha publicado en otras secciones como Sociedad o Economía.

Los años 1996 y 2003 destacan en este diario por el mayor número de informaciones jurídicas publicadas, y además ambos coinciden con este número. En 1996 destacan las informaciones relacionadas con el "caso Nécora", mientras que en 2003 se publican noticias como el procesamiento a Bin Laden y otros miembros de Al Qaeda por el juez Garzón, y noticias sobre el caso Wanninkhof.

En $A b c$ se muestra mayor información jurídica en las secciones España/Nacional e Internacional. Al contrario que El País, donde existen más años en los que no se ha publicado. El año 1998 más destacable por el número 
de informaciones, mientras que en El País en este año no se ha publicado información alguna.

Cabe hacer hincapié en el número de páginas, en $A b c$ mayor que en El País.

Ambos periódicos generalistas coinciden en que la información jurídico-política en las secciones España/Nacional e Internacional es la que más relevancia tiene por el número de informaciones jurídicas que se publican con respecto a otras secciones como Sociedad y Economía.

\subsubsection{La información jurídico-política en la prensa económica a través de Cinco Días (1981-2011)}

El periódico Cinco Días, al tratarse de un diario económico, cuenta con otras secciones diferentes a las de los periódicos generalistas, tal y como se ha visto en puntos anteriores. Es cierto que Cinco Días tenía una sección llamada Política donde se incluían este tipo de informaciones, pero esta solo estuvo presente hasta 1993. Al igual que las secciones Nacional e Internacional que solo están presentes en los primeros años de la década de los ochenta. Por este motivo, estas informaciones jurídico-políticas pasaron a publicarse en otro tipo de secciones, tales como Tribunales y Gestión.

Parece ser que últimamente estas informaciones se incluyen en la sección Empresas, la cual tiene el mayor número de informaciones jurídicas analizadas en el periódico económico. Para representar estos datos que nos ofrece Cinco Días se deben incluir las secciones del periódico a las que se ha hecho referencia: Política, Nacional, Internacional, Tribunales, Gestión y Empresas.

\begin{tabular}{|c|c|c|}
\hline Años & $\begin{array}{l}\text { Frecuencia (Información Juridica } \\
\text { Politica/Nacional//nternacional/Tribunales/ } \\
\text { Gestión/Empresas) }\end{array}$ & $\begin{array}{l}\text { Porcentaje } \\
(\%)\end{array}$ \\
\hline 1981 & 1 & 2,33 \\
\hline 1982 & 1 & 2,33 \\
\hline 1983 & 1 & 2,33 \\
\hline 1984 & 2 & 4,65 \\
\hline 1985 & 0 & 0,00 \\
\hline 1986 & 0 & 0,00 \\
\hline 1987 & 1 & 2,33 \\
\hline 1988 & 0 & 0,00 \\
\hline 1989 & 1 & 2,33 \\
\hline 1990 & 0 & 0,00 \\
\hline 1991 & 1 & 2,33 \\
\hline 1992 & 1 & 2,33 \\
\hline 1993 & 1 & 2,33 \\
\hline 1994 & 0 & 0,00 \\
\hline 1995 & 3 & 6,98 \\
\hline 1996 & 0 & 0,00 \\
\hline 1997 & 3 & 6,98 \\
\hline 1998 & 6 & 13,95 \\
\hline 1999 & 0 & 0,00 \\
\hline 2000 & 3 & 6,98 \\
\hline 2001 & 4 & 9,30 \\
\hline 2002 & 1 & 2,33 \\
\hline 2003 & 2 & 4,65 \\
\hline 2004 & 2 & 4,65 \\
\hline 2005 & 1 & 2,33 \\
\hline 2006 & 0 & 0,00 \\
\hline 2007 & 1 & 2,33 \\
\hline 2008 & 2 & 4,65 \\
\hline 2009 & 1 & 2,33 \\
\hline 2010 & 2 & 4,65 \\
\hline 2011 & 2 & 4,65 \\
\hline \multicolumn{2}{|c|}{ Total: 43 informaciones juridicas } & 100 \\
\hline
\end{tabular}

La información jurídico-politica en las secciones de

Politica/NacionaV/InternacionaVTribunales/Gestión/Empresas en Cinco Dias (1981-2011).

Fuente: Elaboración propia.

En la tabla aparecen los datos obtenidos de las informaciones jurídicas incluidas en las secciones de Política, Nacional, Internacional, Tribunales, Gestión y Empresas. Aunque en esta tabla los datos aparecen como la 
suma de las informaciones jurídico-políticas de todas estas secciones por años, en la siguiente representación gráfica se distinguen estas secciones con el número de informaciones por año correspondientes.

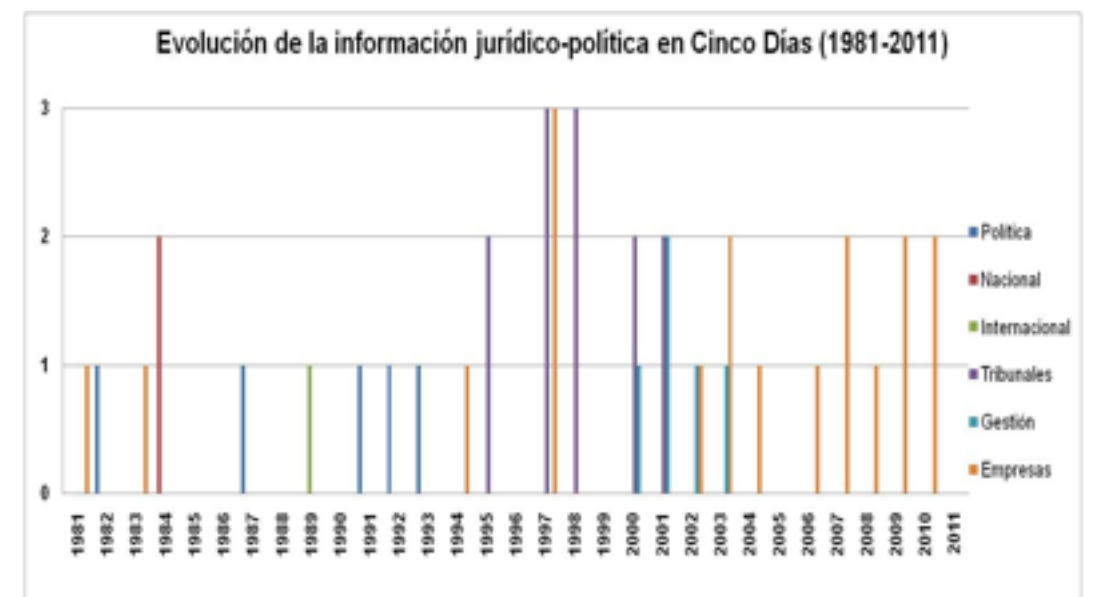

Evolución de la información jurídico-politica en las secciones de

Política/Nacional/Internacional/Tribunales/Gestión/Empresas en Cinco Dias (1981-2011).

Fuente: Elaboración propia.

La información jurídico-política parece ser que en los últimos años se decante por incluirse en la sección Empresas, así lo muestra el gráfico. Aunque cabe destacar que en esta sección también aparecen informaciones jurídicas de otra índole.

Se puede decir que la información jurídico-política en la prensa económica queda un poco repartida en las secciones representadas, pero en ellas también se publica información jurídica sobre otras áreas que no son referentes a la política, sobre todo las que tienen que ver con economía.

Por todo esto, no se puede hablar de una sección fija en la prensa económica para mostrar la información jurídico-política, a diferencia de la prensa generalista, que como se ha comprobado incluye este tipo de información en las secciones Nacional/España e Internacional.

La información jurídico-política en los periódicos generalistas no ha cambiado mucho, es decir, que esta clase de información continúa publicándose en las secciones de España/Nacional e Internacional. La diferencia con el periódico económico es que actualmente en Cinco Días ya no existen las secciones Nacional, Internacional y Política, por este motivo la información jurídica en general se centra en otras secciones como son Empresas, Tribunales y Gestión.

El diario económico es el que más ha evolucionado con respecto a las secciones que incluyen este tipo de información, que han estado en constante cambio frente a los periódicos generalistas, que han sido en este aspecto más conservadores.

Queda constatado, que la información jurídico-política aparece principalmente en los diarios generalistas, siendo el económico el que menos información de este tipo aporta, puesto que se centra más en otras noticias. Cabe destacar que no tiene una sección fija y que esta información suele centrarse en secciones como Nacional/España e Internacional, siendo el periódico especializado una excepción por incluir otro tipo de secciones, tales como empresas, gestión, etc., entre otras.

\section{CONCLUSIONES}

Las conclusiones a las que se han llegado son las siguientes:

1. El periodismo jurídico no solo corresponde a la información de sucesos y de tribunales, sino que se mueve en todas las áreas de un periódico, aparece en la información política, económica, de deportes, de sociedad, de gente famosa; y se mueve en los ámbitos de la justicia, los tribunales, los sucesos, las sentencias, las leyes y los delitos. 
2. En los últimos años el periodismo jurídico ha experimentado uno de los crecimientos mayores de su historia. No es difícil encontrarnos con que los periódicos abren portadas con este tipo de información, así como también la apertura de informativos en radios y televisiones.

3. La prensa especializada en economía dedica un espacio específico a la información jurídica, incluyendo también como información jurídica la relacionada con despachos de abogados, colegio de procuradores, etc. Además contiene secciones, suplementos o revistas jurídicas que aportan sentencias actuales, entrevistas a gente del mundo jurídico u opiniones de jueces y magistrados sobre un tema jurídico de actualidad concreto. A diferencia de la prensa generalista, que carece de una sección fija para la información jurídica, así como de suplementos o revistas sobre la misma.

4. En las secciones donde más información jurídica aparece son las referentes a Nacional/España en el caso de los diarios generalistas. En los periódicos especializados en economía, es la sección empresas la que más información jurídica contiene.

\section{REFERENCIAS BIBLIOGRÁFÍCAS Y HEMEROGRÁFICAS}

\section{Libros}

BERELSON, B., (1984). Content Análisis in Communications Research. Nueva York: Uni. Press.

GALLEGO, J., (2003). Información de sociedad: del suceso a la calidad de vida. Barcelona: Col.lecció Materials, Universitat Autònoma de Barcelona, Servei dePublicacions.

HERNÁNDEZ, R., FERNÁNDEZ, C. and BAPTISTA, P., (2007). Fundamentos de metodología de la investigación. Madrid: McGraw-Hill.

PIÑUEL, J.L. y GAITÁN, J.A., (1995). Metodología general. Conocimiento científico e investigación en la comunicación social. Madrid: Síntesis.

QUESADA, M., (2007). Periodismo de sucesos. Madrid: Editorial Síntesis.

RONDA, J. and CALERO, J.M., (2000). Manual de Periodismo Judicial. Sevilla: Universidad de Sevilla.

\section{Capítulos de libros}

POU, M.J.: Periodismo jurídico. En CEBRIÁN, B. y MIRÓN, L. M. (Coords.) (2013): Áreas del periodismo. Salamanca: Comunicación Social, pp. 133.

QUESADA, M.: Los sucesos como foco de atención informativa. En CAMACHO, I. (Coord.) (2010): La especialización en el periodismo. Sevilla/Zamora:Comunicación Social, pp. 58.

\section{Artículos de revistas}

DE FONTCUBERTA, M. “El discurso de la prensa del corazón”, Revista Análisi, n 13, (1990).

MERCADO, M.T. “La información del corazón en televisión”. Revista Latina de Comunicación Social, $n^{\circ} 21$, (1999).

RONDA, J., "El periodismo judicial en España". Revista Ámbitos, nº especial 9-10, (Segundo Semestre 2002Año 2003), pp. 187.

\section{Diarios. Consulta de los diarios Abc, El País y Cinco Días en las siguientes hemerotecas:}

- Hemeroteca Municipal de Madrid

- Hemeroteca Municipal de Valencia 
- Hemeroteca de la Universidad CEU Cardenal Herrera

- Hemeroteca del diario Abc en su página web: http://hemeroteca.abc.es/

(1) Creación de la Constitución de 1978 y cómo se estructuran los poderes. Esclarecimiento obtenido de la entrevista realizada a Gabriela Bravo, portavoz del Consejo General del Poder Judicial.

(2) Publicidad de los juicios e interés social por ellos. Explicación sacada de una entrevista realizada a Gabriela Bravo, portavoz del Consejo General del Poder Judicial.

(3) Conclusión sacada de la entrevista realizada a Gabriela Bravo, portavoz del Consejo General del Poder Judicial. En ella, acentúa que mientras existe la etapa democrática en España la información jurídica es relevante porque la Constitución creó un verdadero Poder Judicial inexistente hasta entonces, donde su función despierta el interés de los medios de comunicación.

\section{Breve semblanza de la autora}

María José Antich Gil es licenciada en periodismo y Doctora en Comunicación. Ha desempeñado su trayectoria académica en la Universidad Cardenal Herrera CEU de Valencia. Ha trabajado como becaria en diferentes medios de comunicación, radio y televisión. En su tesis se ha especializado en periodismo jurídico. Actualmente colabora como socia en asociaciones de periodistas, impartiendo cursos y organizando mesas redondas, para aportar conocimiento sobre el tema.

Ámbitos. Revista Internacional de Comunicación, n.26, año 2014, tercer trimestre (otoño). Recibido: 19/6/2014

Aprobado: 1/8/2014 